\title{
Implementing Consensus Based Distributed Control in Power System Toolbox
}

\author{
Minyue Ma and Lingling Fan \\ Department of Electrical Engineering \\ University of South Florida \\ Tampa, FL 33620 \\ url: http://power.eng.usf.edu \\ Email: linglingfan@usf.edu
}

\begin{abstract}
This paper proposes a distributed control algorithm which is based on consensus and subgradient method. According this algorithm, each agent in a power system network can exchange information with their direct connected neighbors to unify all local marginal prices by consensus method. Synchronously, all agents update price information and power demands information to do economic operation based on subgradient method. The proposed method can realize economic dispatch and frequency regulation simultaneously. Power system tool box (PST) is selected as the platform to implement the distributed control algorithm. The approach to implement this algorithm in PST is presented. Effect of the proposed algorithm is compared with a secondary frequency control via simulation results.
\end{abstract}

Index Terms-Consensus method, subgradient method, economic dispatch, frequency regulation, power system tool box.

\section{INTRODUCTION}

The objective of this paper is to propose and implement a distributed control algorithm based on consensus and subgradient method. According this algorithm, each agent in a power system network can exchange information with their direct connected neighbors to unify all local marginal prices by consensus method. Synchronously, all agents update price information and power demands information to do economic operation based on subgradient method. The proposed method can realize economic dispatch and frequency regulation simultaneously. PST is selected as the platform to implement the distributed control algorithm. The approach to implement this algorithm in PST is presented. Effect of the proposed algorithm is compared with a secondary frequency control via simulation results.

The rest of the paper is organized as follows. Section II describes the consensus subgradient algorithm. Section III presents PST implementation techniques. Section IV presents case study results and Section V concludes the paper.

\section{Consensus Subgradient Algorithm}

\section{A. Consensus Algorithm}

Consensus algorithm has been applied in a power system network to solve distributed optimization problems [1]. As an interaction rule which regulate information exchange between an agent and all of the neighbours, the purpose of this algorithm is to make information in different agent converge to a single value [1], [2]. To reach this purpose, the information discovery process in a agent can be described by a discrete time linear system as follows [3].

$$
X^{k+1}=A X^{k}
$$

where $X_{i}$ are the state information that collected in agent $i ; k$ means the $k^{t h}$ iteration step; $A \in \mathbf{R}^{n \times n}$ is a stochastic matrix.

A stochastic matrix has following characteristics:

- It is a square matrix.

- All elements of this matrix are nonegative real numbers.

- The elements sums for each row is one.

Using the stochastic matrix in the iteration means $X_{i}$ is an weighted average value of all $X_{j}(j=1, \cdots, n)$. As the designation of $A$ relates with the convergence speed of $X_{i}[\overline{3}]$, it is necessary to use a specify method to configure the matrix $A$. In this paper, the Metropolis method [3], [4] is applied to design $A$, which can be described as:

$$
a_{i j}= \begin{cases}\frac{1}{\max \left(n_{i}, n_{j}\right)+1} & j \in N_{i} \\ 1-\sum_{j \in N_{i}} \frac{1}{\max \left(n_{i}, n_{j}\right)+1} & j=i \\ 0 & \text { otherwise }\end{cases}
$$

where $a_{i j}$ is an element of $A$ which position is the $i^{t h}$ row and $j^{\text {th }}$ column; $N_{i}$ is the set of the neighboring agents for agent $i$; $n_{i}$ and $n_{j}$ respectively are the numbers of agents which directly connect with agent $i$ and $j$.

\section{B. Subgradient Update}

Subgradient update method is an iterative method which is wildly used to solve optimization problems iteratively. For a three-area three-machine system in Fig. 11, the corresponding economic dispatch problem can be expressed as:

$$
\begin{array}{cl}
\min _{P_{i}} & C_{1}\left(P_{1}\right)+C_{2}\left(P_{2}\right)+C_{3}\left(P_{3}\right) \\
\text { subject to: } & P_{1}+P_{2}+P_{3}=D_{1}+D_{2}+D_{3}
\end{array}
$$

where $P_{i}(i=1,2,3)$ is the local generator dispatch of area $\mathrm{i}$, and $D_{i}(i=1,2,3)$ is the local power demand; $C\left(P_{i}\right)=$ $a_{i} P_{i}^{2}+b_{i} P_{i}+c_{i}$ is the cost function. 


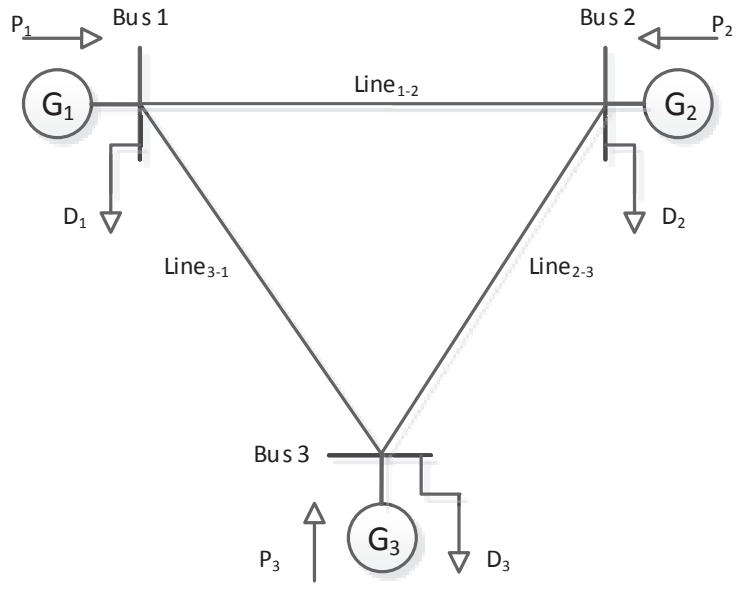

Fig. 1. The test system. is:

Basing on (3), a Lagrange function can be obtained, which

$$
\begin{array}{r}
L\left(P_{i}, \lambda\right)=C_{1}\left(P_{1}\right)+C_{2}\left(P_{2}\right)+C_{3}\left(P_{3}\right) \\
+\lambda\left(D_{1}+D_{2}+D_{3}-P_{1}-P_{2}-P_{3}\right)
\end{array}
$$

where $\lambda_{i}$ is the price of each area.

According (3) and (4), the dual problem of (3) can be expressed as:

$$
\max _{\lambda}\left\{\min _{P_{i}} L\left(P_{i}, \lambda\right)\right\}
$$

To solve this dual problem, the $\lambda$ update process is as follows.

$$
\lambda^{k+1}=\lambda^{k}+\alpha\left(D_{1}+D_{2}+D_{3}-P_{1}^{k}-P_{2}^{k}-P_{3}^{k}\right) .
$$

The partial derivative of $L\left(P_{i}, \lambda\right)$ about each $P_{i}$ should be zero, if the objective function reach the minimum value. Thus, a equation which describe the relation of $P_{i}$ and $\lambda$ can be obtained:

$$
\lambda=2 a_{i} P_{i}+b_{i} \Rightarrow P_{i}=\frac{\lambda-b_{i}}{2 a_{i}} .
$$

Combining (6) and (7), an iteration process to find the economic dispatch of $P_{i}$ is obtained:

$$
\left\{\begin{array}{l}
P_{i}^{k}=\frac{\lambda^{k}-b_{i}}{2 a_{i}} \\
\lambda^{k+1}=\lambda^{k}+\alpha\left(D_{1}+D_{2}+D_{3}-P_{1}^{k}-P_{2}^{k}-P_{3}^{k}\right) .
\end{array}\right.
$$

where $\alpha>0$ is the step size.

a) Convergence discussion: In (8), the value of $\alpha$ is important for $\lambda$ convergence. Combining the equations in (8), the update procedure of $\lambda$ can be expressed as:

$$
\begin{gathered}
\lambda^{k+1}=\left[1-\alpha\left(\frac{1}{2 a_{1}}+\frac{1}{2 a_{2}}+\frac{1}{2 a_{3}}\right)\right] \lambda^{k} \\
+\alpha\left(D_{1}+D_{2}+D_{3}-\frac{b_{1}}{2 a_{1}}-\frac{b_{2}}{2 a_{2}}-\frac{b_{3}}{2 a_{3}}\right) .
\end{gathered}
$$

According numeric analysis, to ensure convergence of $\lambda$, the value of $\alpha$ should satisfy the following constraint:

$$
\begin{array}{r}
\left|1-\alpha\left(\frac{1}{2 a_{1}}+\frac{1}{2 a_{2}}+\frac{1}{2 a_{3}}\right)\right|<1 \\
\Rightarrow 0<\alpha<\frac{\frac{1}{2 a_{1}}+\frac{1}{2 a_{2}}+\frac{1}{2 a_{3}}}{}
\end{array}
$$

Basing on (9), to get the fastest converge speed of $\lambda$, the $\alpha$ can be set to be:

$$
\alpha=\frac{1}{2 a_{1}}+\frac{1}{2 a_{2}}+\frac{1}{2 a_{3}} .
$$

For example, if $a_{1}=1, a_{2}=2, a_{3}=3$, the limit of $\alpha$ is: $0<\alpha<2.1818$, and "the fastest converge $\alpha$ " is: $\alpha=1.0909$.

The $\alpha$ setting in $(11)$ is only based on iteration function, so it might cause stability in real systems [5]. Thus, the calculation result of (11) should be used with care.

\section{Consensus subgradient method}

The power imbalance of a system is related to frequency deviation, which can be expressed as:

$$
\Delta P=-K \Delta f .
$$

where $K$ is related to droop parameters.

Thus, the subgradient term: $\alpha\left(D_{1}+D_{2}+D_{3}-P_{1}^{k}-P_{2}^{k}-P_{3}^{k}\right)$ can be consider as the frequency compensation. It means that applying iteration process in (8) and making $P_{i}^{k}$ as the turbine governor reference power $\left(P_{\text {refi }}\right)$ has the potential to reach economic dispatch and frequency regulation simultaneously.

Combining the consensus and subgradient update method, we have the following update:

$$
\lambda^{k+1}=A \lambda^{k}+\alpha\left(D_{1}+D_{2}+D_{3}-P_{1}^{k}-P_{2}^{k}-P_{3}^{k}\right) .
$$

For the system in Fig. 1, and use the method in (2), the matrix A is:

$$
A=\left[\begin{array}{lll}
\frac{1}{3} & \frac{1}{3} & \frac{1}{3} \\
\frac{1}{3} & \frac{1}{3} & \frac{1}{3} \\
\frac{1}{3} & \frac{1}{3} & \frac{1}{3}
\end{array}\right]
$$

As the consensus algorithm will guarantee $\lambda$ to the same in each agent. The following iteration process can make the system achieve economic dispatch and frequency regulation simultaneously.

$$
\left\{\begin{array}{l}
P_{i}^{k}=\frac{\lambda^{k}-b_{i}}{2 a_{i}} \\
\lambda^{k+1}=A \lambda^{k}+\alpha\left(D_{1}+D_{2}+D_{3}-P_{1}^{k}-P_{2}^{k}-P_{3}^{k}\right) .
\end{array}\right.
$$

\section{Power System ToOlbox IMPlementation}

The Power System Toolbox (PST) is a MAtlab-based package which is mainly utilized to provide power system dynamics simulation and control design [6]. Its working principle, in simply term, is coding components of power system to MATLAB functions base on the mathematic models of these components, and running functions in certain sequence to display the simulation result of the power system which include these components. 
For each function, the fundamental structure [6] can be expressed as follow:

$$
\begin{aligned}
\dot{x} & =f(x, u) \\
y & =g(x, u)
\end{aligned}
$$

where $x$ is the vector of state variables; $u$ is the vector of input variables; $y$ is the output varaibles.

Generally, in a simulation process of PST, functions should run three steps:

- Step 1: Initialzation of the state variables (It is marked as flag $=0$ ). In this step, all state variables $x$ will be set to initial values according to the parameters which is defined in a data file. This step only run one time at $t=0$.

- Step 2: Network interface computation (It is marked as flag $=1$ ). In this step, the interface variables $y$ will be calculated based on current system state. This step will run at every time step which is marked as the $k^{\text {th }}$ step.

- Step 3: Dynamic calculation (It is marked as flag=2). In this step, the system state variables will be calculated based on current system state and their change rate at the $k^{t h}$ step. Moreover, the change rate of state variables for next time step(the $k+1^{t h}$ ) will be calculated. The step 3 will run at every time step.

An important advantage of PST is that it is open source and it is easy to be modified by user. As its models are totally built in equation and matrix forms, the structure and scale of a PST system model can be easily changed. Moreover, adding a new function is not complicated after following some simple rules.

\section{A. PST Implementation: How to change turbine's reference signal}

In this paper, to implement the proposed consensus subgradient algorithm in PST, the function: $\mathrm{mtg}$ _sig.m is modified. The basic purpose of mtg_sig.m function is to determine the changed value of turbine governor reference power. The turbine governor reference power is marked as $P_{\text {refi }}$ in Fig. 2 .

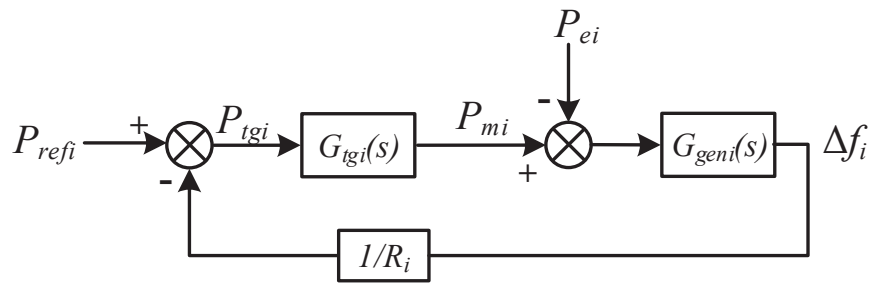

Fig. 2. Generator turbine-governor control block (Droop control only).

For the control block in Fig. 2. more detailed description is presented in Section IV.

To apply the proposed method, the equation group 15 should be used to calculate $P_{\text {refi. }}$. So a section of code which is based on equation group (15) is added into mtg_sig.m.

\section{B. How to implement discrete distributed algorithm}

As in a real system, agents exchange their information every several seconds, the variable update in $\mathrm{mtg}$ _sig.m should be discrete and the time step length is a few seconds. However, generally, the global time step length for numerical integration is much smaller than one second. Otherwise it can not ensure the accuracy of dynamic simulation of the system. To achieve this discrete data update in special time step length, a section of code is added into mtg_sig.m, and its flow cart is shown in Fig. 3

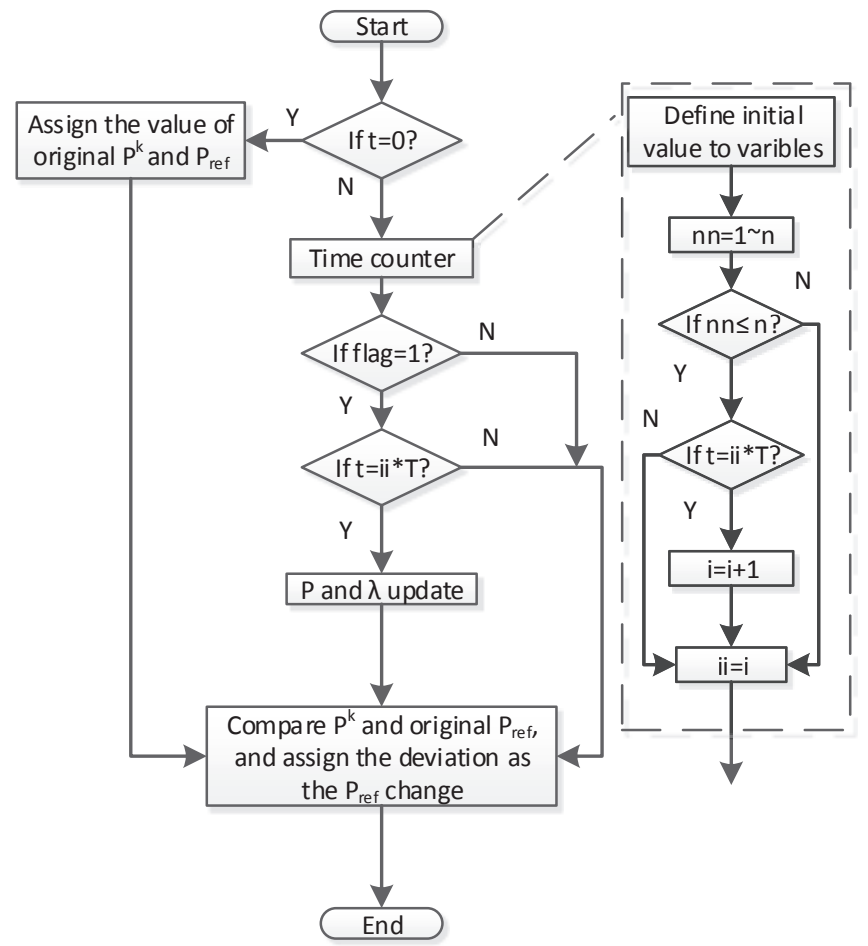

Fig. 3. Discrete update. Time interval is $\mathrm{T}$

Using the modfied mtg_sig.m which is described in above sections, a simulation system model including the proposed distributed control algorithm can be implemented in PST platform.

\section{Simulation Results}

The test system is a 3-area 3-machine system shown in Fig. 1. The system parameters are displayed in Table. I and Table. III. For per unit value, the system power base is $100 \mathrm{MW}$, and frequency base is $60 \mathrm{HZ}$. Based on Fig. 1, the digital subscript of parameters in these tables are corresponding to the bus and line number. The system model has been built in PST.

Assume the cost functions of machine 1,2 and 3 are:

$$
\begin{gathered}
C_{1}\left(P_{1}\right)=P_{1}^{2}+2 P_{1} \\
C_{2}\left(P_{2}\right)=2 P_{2}^{2}+2 P_{2} \\
C_{3}\left(P_{3}\right)=3 P_{3}^{2}+2 P_{3} .
\end{gathered}
$$

Each generator has a turbine governor and a primary droop control. The control block diagram is shown in Fig. 2, and the transfer function of the turbine governer is as follows:

$$
G_{\mathrm{tgi}}(s)=\frac{1}{1+s T_{s}} \frac{1+s T_{3}}{1+s T_{c}} \frac{1+s T_{4}}{1+s T_{5}}
$$


TABLE I

Bus CONFIGURATIONS.

\begin{tabular}{c|c|c}
\hline Name & Value(unit) & Description \\
\hline$V_{1}$ & $1.04(\mathrm{pu})$ & initial voltage \\
$V_{2}$ & $1.025(\mathrm{pu})$ & magnitude of each \\
$V_{3}$ & $1.025(\mathrm{pu})$ & bus \\
\hline$\theta_{1}$ & $0.0($ degree$)$ & initial voltage \\
$\theta_{2}$ & $9.3($ degree) & angle of each \\
$\theta_{3}$ & $4.7($ degree $)$ & bus \\
\hline$P_{1}$ & $0.716(\mathrm{pu})$ & inital active \\
$P_{2}$ & $1.63(\mathrm{pu})$ & power that inject \\
$P_{3}$ & $0.85(\mathrm{pu})$ & to each bus \\
\hline$Q_{1}$ & $0.27(\mathrm{pu})$ & initial reactive \\
$Q_{2}$ & $0.067(\mathrm{pu})$ & power that inject \\
$Q_{3}$ & $-0.109(\mathrm{pu})$ & to each bus \\
\hline$D_{P 1}$ & $1.25(\mathrm{pu})$ & active power \\
$D_{P 2}$ & $0.90(\mathrm{pu})$ & demand on each \\
$D_{P 3}$ & $1.00(\mathrm{pu})$ & bus \\
\hline$D_{Q 1}$ & $0.5(\mathrm{pu})$ & reactive power \\
$D_{Q 2}$ & $0.3(\mathrm{pu})$ & demand on each \\
$D_{Q 3}$ & $0.35(\mathrm{pu})$ & bus \\
\hline
\end{tabular}

TABLE II

TRANSMission LiNE CONFIGURATIONS.

\begin{tabular}{c|c|c}
\hline Name & Value(unit) & Description \\
\hline$R_{1-2}$ & $0.042(\mathrm{pu})$ & The equivalent \\
$R_{2-3}$ & $0.0204(\mathrm{pu})$ & resistance of each \\
$R_{3-1}$ & $0.056(\mathrm{pu})$ & line \\
\hline$X_{1-2}$ & $0.3661(\mathrm{pu})$ & The equivalnet \\
$X_{2-3}$ & $0.2939(\mathrm{pu})$ & reactance of each \\
$X_{2-1}$ & $0.3782(\mathrm{pu})$ & line \\
\hline$C_{1-2}$ & $0.5445(\mathrm{pu})$ & The equivalent \\
$C_{2-3}$ & $0.358(\mathrm{pu})$ & charging of each \\
$C_{3-1}$ & $0.516(\mathrm{pu})$ & bus \\
\hline \multicolumn{2}{|c}{} \\
\hline
\end{tabular}

where the $T_{s}$ is servo time constant; $T_{c}$ is HP turbine time constant; $T_{c}$ is HP turbine time constant; $T_{3}$ is transient gain time constant; $T_{4}$ is time constant to set HP ratio; $T_{5}$ is reheater time constant. And the configuration of these number for each $G_{\mathrm{tgi}}(s)$ is listed in Table. III. The subscripts of $G_{\mathrm{tgi}}(s)$ are corresponding with the generator number.

For this experiment, at $t=40 \mathrm{~s}$, loads will increase: $D_{1}$ increases $0.3 ; D_{2}$ increase $0.1 ; D_{3}$ increase 0.2. Total load increase is $0.6 \mathrm{pu}$. The proposed method will be compared with droop control only case and secondary control case to demonstrate its advantage.

\section{A. Case 1-Droop control only}

In this case, The system work in original condition, which only has droop control.

Fig. 4 presents the system response for 100 seconds in case 1 . After the load increase, the power increasing is nearly evenly shared among three generators. This is caused by

TABLE III

PARAMETERs OF TURbine Governors.

\begin{tabular}{c|ccccc}
\hline PG number & $T_{s}$ & $T_{c}$ & $T_{3}$ & $T_{4}$ & $T_{5}$ \\
\hline$G_{t g 1}(s)$ & 0.45 & 0.1 & 0.0 & 1.25 & 5.0 \\
\hline$G_{t g 2}(s)$ & 0.5 & 0.5 & 0.0 & 1.25 & 5.0 \\
\hline$G_{t g 3}(s)$ & 0.24 & 0.18 & 0.0 & 1.25 & 5.0 \\
\hline
\end{tabular}

the similar droop constants in three generators $\left(\frac{1}{R_{1}}=25.1\right.$, $\frac{1}{R_{2}}=25.5, \frac{1}{R_{3}}=25.4$ ). The system frequency is lower than the nominal frequency. According to (12, this deviation is caused by power imbalance between $P_{\text {refi }}$ and $P_{m i}$. In this case, $K=\Sigma \frac{1}{R_{i}}=\frac{1}{R_{1}}+\frac{1}{R_{2}}+\frac{1}{R_{3}}=76, \Delta P=P_{\text {refi }}-P_{m i}=$ -0.6060615 , so $\Delta f=-\frac{\Delta P}{\Sigma \frac{1}{R_{i}}} \approx 0.0079745$, which is the same with the value that obtained from simulation results.
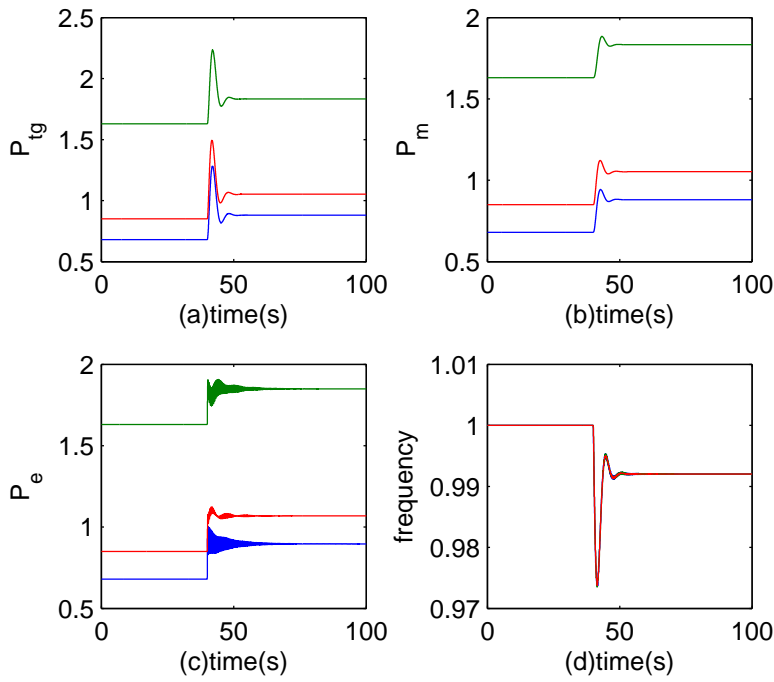

Fig. 4. Droop control only system response. (a) Turbine governor per unit input power on system base; (b) Turbine governor per unit output mechnical power on system base; (c) Generators'output per unit electric power on system base; (d) Generators' per unit frequency on system base.

\section{B. Case 2-secondary frequency control}

A secondary frequency control is added on the system, the control block is shown in Fig. 5. $K_{1}=40, K_{2}=20, K_{2}=$ 13.3333 .

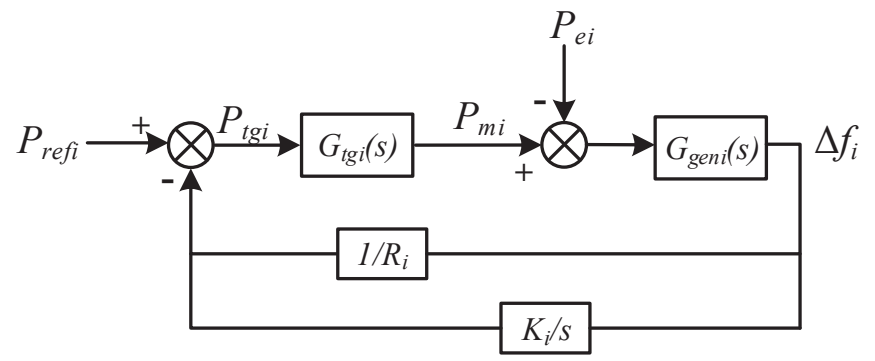

Fig. 5. Secondary frequency control added control block.

Fig. 6 presents the system response of case 2. It can bee seen that as the secondary frequency control is used on system, the system frequency is restored to nominal frequency after load increased. Moreover, the power sharing on each generator is inversely proportional $(3: 2: 1)$ with the quadratic coefficients $(1: 2: 3)$ of cost functions. It is due to the modified integral gains. However, the secondary control only response for system state change, thus it can not make the system achieve economic dispatch. 

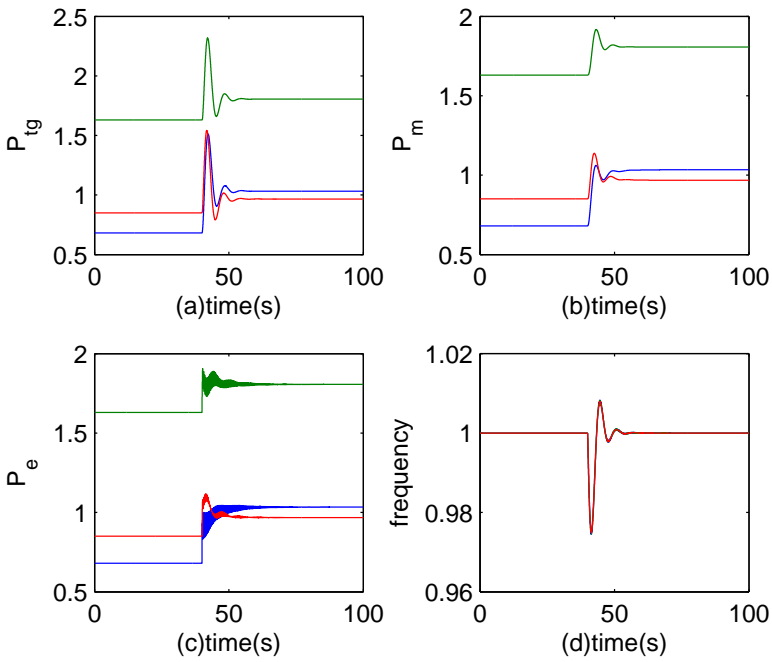

Fig. 6. Secondary frequency control added on system. (a) Turbine governor per unit input power on system base; (b) Turbine governor per unit output mechnical power on system base; (c) Generators'output per unit electric power on system base; (d) Generators' per unit frequeny on system base.

\section{Case 3 - consensus subgradient update method}

The proposed consensus subgradient method is implemented on the system. The update process start from the 2 second, and the time step length is 2 seconds. The control block is shown in Fig. 7. $\alpha=0.5$.

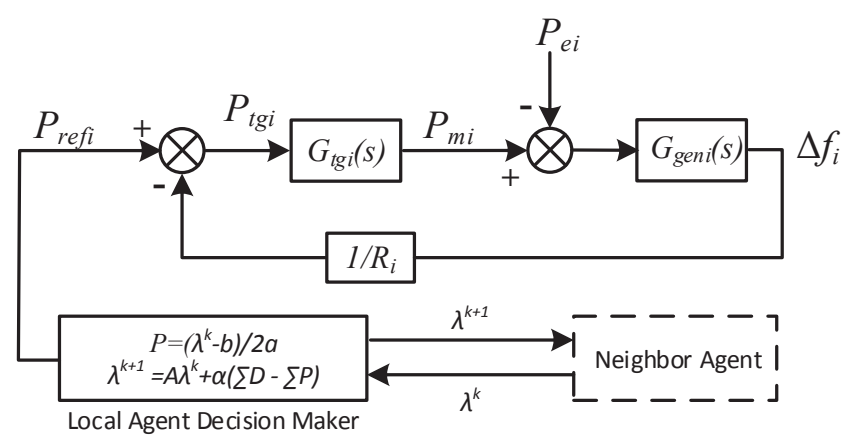

Fig. 7. Consensus Subgradient algorithm implemented block.

Fig. 8 presents the system response while the consensus method has been applied. In this case, the generaotrs' active power output is inversly propotional to the cost functions quadratic coefficients. The system frequency is lower than nominal frequency but the deviation: $\Delta f \approx 0.00054351$ is smaller than $\Delta f \approx 0.0079745$ which in case 1 . Considering equation (12) again, in case $3, K=76$, so power imbalance can be caculated: $\Delta P=-K \Delta f \approx-0.0413068$. And that is the same with simulation result. Even though it disturbed frequency regulation, this power imbalance is acceptable because the loss of system is not considered in the subgradient $\operatorname{term}\left(D_{1}+D_{2}+D_{3}-P_{1}^{k}-P_{2}^{k}-P_{3}^{k}\right)$.

Fig. 9 presents the iteration steps of $\lambda$ and $P_{\text {ref }}$. With consensus method the $\lambda$ is converged to a uniform value, and $P_{\text {ref }}$ is changed to reach the economic dispatch.
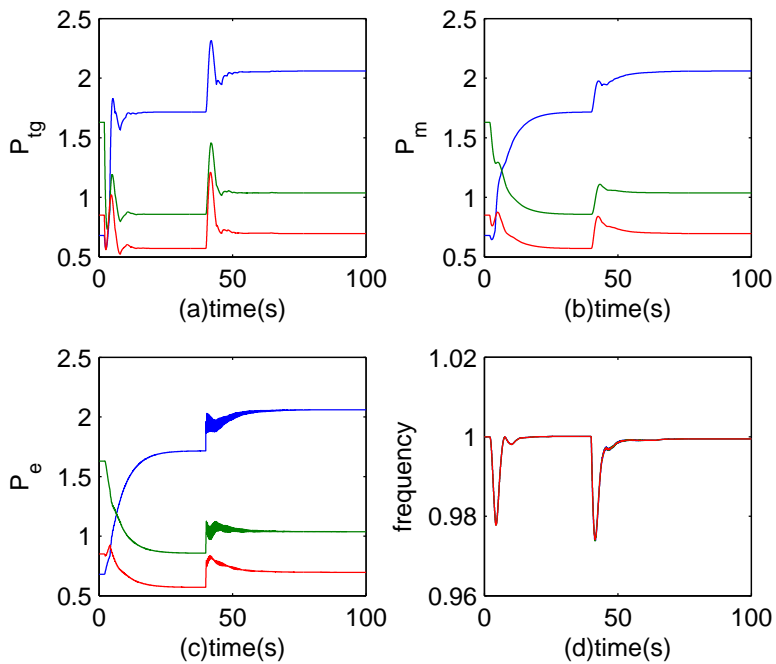

Fig. 8. Consensus Subgradient is used on system. (a) Turbine governor per unit input power on system base; (b) Turbine governor per unit output mechnical power on system base; (c) Generators'output per unit electric power on system base; (d) Generators' per unit frequeny on system base.
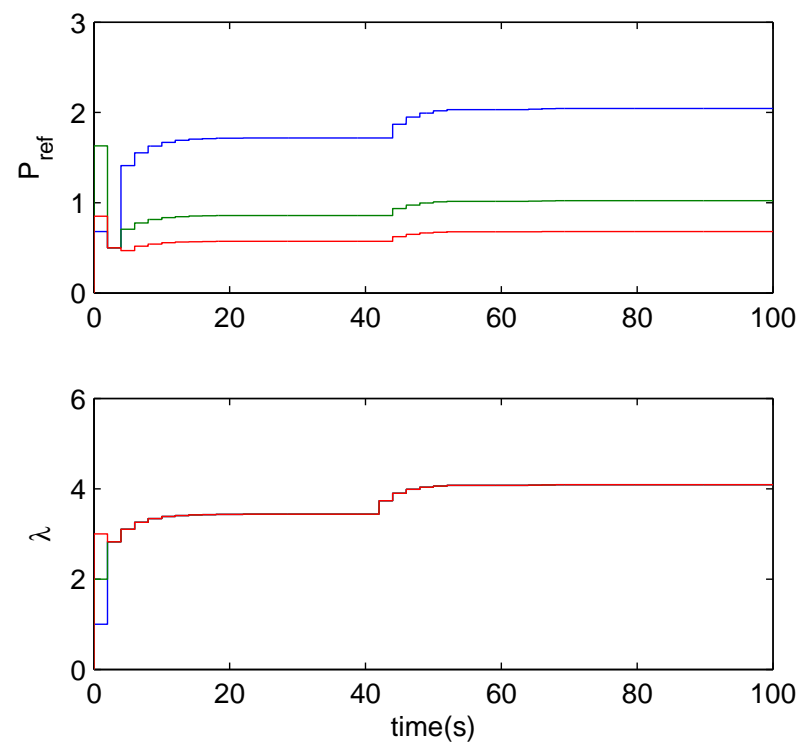

Fig. 9. Iteration process of $P_{\text {ref }}$ and $\lambda$.

In Table. IV] the final value of $P_{e i}$ of each generator in different case is listed, and a comparison for total cost is presented to demonstrate the economic dispatch is achieved through proposed consensus method. Moreover, the column "Comparison" is used to show that even the output of the cheapest generator is higher and the most expensive one is lower than case 3 , the total cost is still higher than case 3 .

\section{D. $\lambda$ Convergence Study}

Fig. 10 to Fig. 13 present the convergence of $\lambda$ for different $\alpha$ base on case 3. From these results, it can be seen that compare with random choice, $\lambda$ converge faster when $\alpha=$ 
TABLE IV

Comparison of $P_{\text {ei }}(p u)$ and Total Cost for Different CASE.

\begin{tabular}{c|c|c|c|c}
\hline Name & case 1 & case 2 & case 3 & Comparison \\
\hline$P_{e 1}$ & 0.8967061 & 1.0336618 & 2.0601324 & 2.2601324 \\
$P_{e 2}$ & 1.8492829 & 1.8064414 & 1.0377092 & 1.0377092 \\
$P_{e 3}$ & 1.0684905 & 0.9677809 & 0.6967247 & 0.4967247 \\
\hline$\Sigma C\left(P_{\mathrm{ei}}\right)$ & 18.6977512 & 18.0204856 & 15.4432347 & 15.5912181 \\
\hline
\end{tabular}

1.0909 which is obtained by equation (11), but the transient of frequency is more serious. Moreover, the requirement of convergence of $\lambda$ that is: $0<\alpha<2.1818$, which is calculated by equation (10), is verified. In Fig. 10,to Fig. 11] time range of $0-20$ seconds is selected to show more detailed convergence process. In Fig. 13, to observe the convergence when $\alpha$ close the marginal value, the time range is extended to 300 senconds.
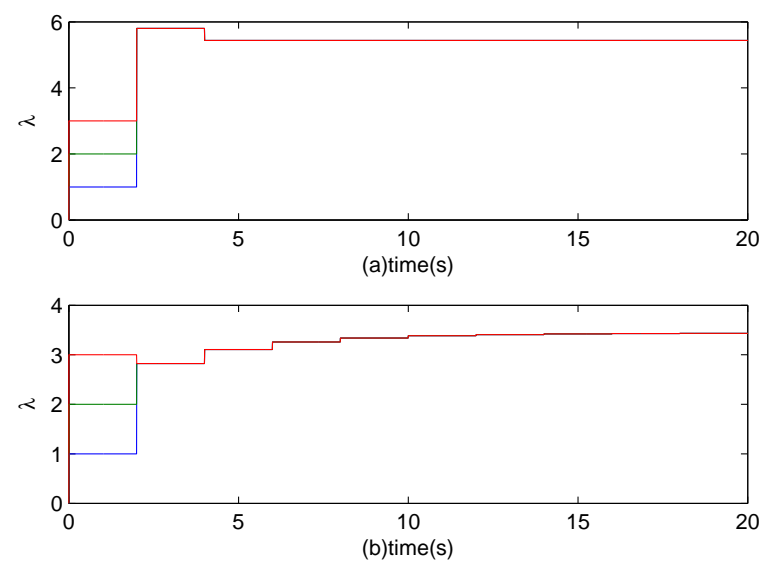

Fig. 10. The convergence of $\lambda$ for different $\alpha$. (a) $\alpha=1.0909$; (b) $\alpha=0.5$

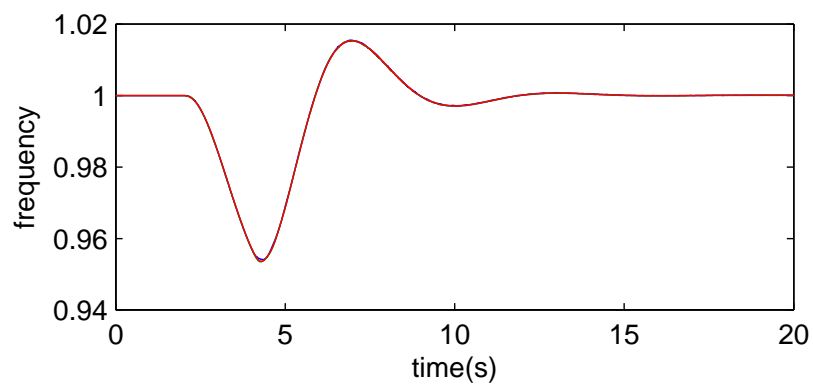

Fig. 11. Generators frequency when $\alpha=1.0909$.

The above results prove that the proposed consensus method can simultaneously deal with economic dispatch and frequency regulation problem.

\section{CONClusion}

In this paper, a consensus subgradient algorithm is proposed for distributed control on power system. This algorithm is capable to make generators in a system achieve economic dispatch and frequency regulation simultaneously. The parameter setting method for iteration process of this algorithm is discussed. And a PST-based simulation model which

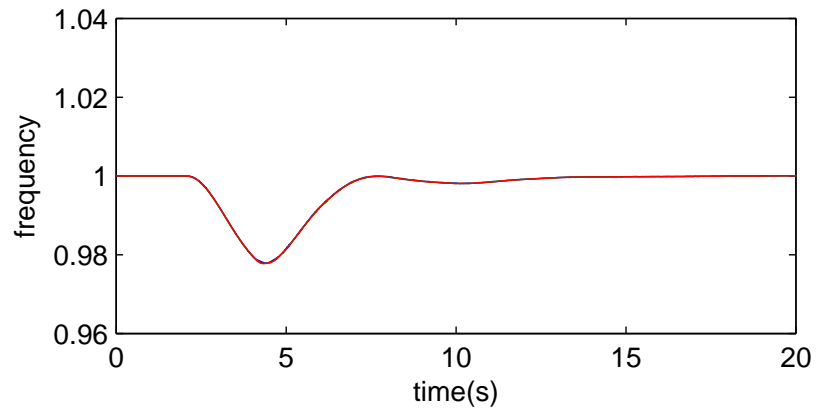

Fig. 12. Generators frequency when $\alpha=0.5$.
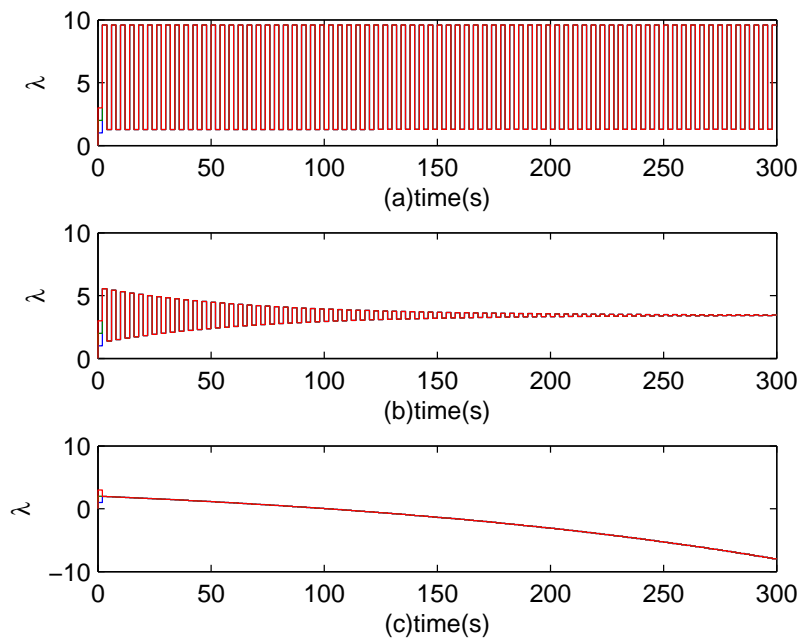

Fig. 13. The convergence of $\lambda$ for different $\alpha$. (a) $\alpha=2.1818$; (b) $\alpha=2.15$; (c) $\alpha=-0.01$.

implemented this algorithm is built. The effect of proposed algorithm is verified by simulation results.

\section{REFERENCES}

[1] M. Yazdanian and A. Mehrizi-Sani, "Distributed control techniques in microgrids," Smart Grid, IEEE Transactions on, vol. 5, no. 6, pp. 29012909, 2014.

[2] R. Olfati-Saber, A. Fax, and R. M. Murray, "Consensus and cooperation in networked multi-agent systems," Proceedings of the IEEE, vol. 95, no. 1, pp. 215-233, 2007.

[3] Y. Xu and W. Liu, "Novel multiagent based load restoration algorithm for microgrids," Smart Grid, IEEE Transactions on, vol. 2, no. 1, pp. 152-161, 2011

[4] L. Xiao, S. Boyd, and S.-J. Kim, "Distributed average consensus with least-mean-square deviation," Journal of Parallel and Distributed Computing, vol. 67, no. 1, pp. 33-46, 2007.

[5] Z. Miao, "Analysis of coupling dynamics for power systems with iterative discrete decision making architectures," submitted to IEEE Transactions on Power Systems.

[6] J. H. Chow and K. W. Cheung, "A toolbox for power system dynamics and control engineering education and research," Power Systems, IEEE Transactions on, vol. 7, no. 4, pp. 1559-1564, 1992. 